\title{
Foundation teaching: a quality improvement programme to improve the quality of structured F1 teaching
}

\author{
Authors: Claire Bradley and Chris Ferguson
}

\begin{abstract}
Aims
Learning and teaching are central tenets of the Foundation Programme Curriculum and the NHS Foundation Programme highlights the need for protected teaching time. We sought to assess and improve the quality of the current structured educational sessions in a single hospital in Northern Ireland.
\end{abstract}

\section{Methods}

We undertook an online survey of foundation year 1 (F1) trainees in our hospital to assess their views on the quality of foundation teaching, especially the existing structured teaching sessions. We specifically asked the F1 doctors to identify any areas for improvement and to propose topics for future teaching. Following the initial online survey, we provided three teaching sessions on targeted areas that trainees had identified, after which we repeated a survey.

\section{Results}

Twelve of 15 doctors responded to the initial survey: the quality of the current structured teaching was rated $73 \%$; the relevance of teaching topics was rated $74 \%$; and $100 \%$ thought the current programme could be improved. Ten of 15 doctors responded to the second survey following three teaching sessions: the quality of the teaching provided was rated $94 \%$; the relevance of teaching topics was rated $98 \% ; 100 \%$ of responders felt the quality of the teaching programme improved following the additional sessions; and $100 \%$ of responders would like continued teaching from trainee doctors.

\section{Conclusion}

Although the existing structured teaching programme was rated highly at the outset by trainees, there was a significant improvement in the trainee ratings for the quality of teaching and the relevance of topics after the addition of trainee-led teaching on topics suggested by foundation doctors. We propose that involving other more senior trainees in F1 teaching would be beneficial and relatively easy to implement. Furthermore, we suggest that involving F1 doctors in the selection of teaching topics is a simple way to improve relevance and quality of teaching.

\section{Conflict of interest statement}

None declared. 